\title{
INTEGRAL observations of Hercules X-1
}

\author{
D. Klochkov ${ }^{1}$, R. Staubert ${ }^{1}$, K. Postnov ${ }^{3}$, N. Shakura ${ }^{3}$, A. Santangelo ${ }^{1}$, S. Tsygankov ${ }^{2}$, A. Lutovinov ${ }^{2}$, \\ I. Kreykenbohm ${ }^{1,4}$, and J. Wilms ${ }^{5}$ \\ 1 Institut für Astronomie und Astrophysik, University of Tübingen, Sand 1, 72076 Tübingen, Germany \\ e-mail: klochkov@astro.uni-tuebingen.de \\ 2 Space Research Institute, Profsoyuznaya str. 84/32, 117997 Moscow, Russia \\ Sternberg Astronomical Institute, Moscow University, 119992 Moscow, Russia \\ 4 INTEGRAL Science Data Centre, Chemin d'Ecogia, 16, 1290, Versoix, Switzerland \\ 5 Dr. Karl Remeis-Sternwarte, Astronomisches Institut, Universität Erlangen-Nürnberg, Sternwartstr. 7, 96049 Bamberg, Germany
}

Received 29 October 2007 / Accepted 29 January 2008

\section{ABSTRACT}

\begin{abstract}
Aims. We investigate the X-ray spectral and timing properties of the accreting X-ray pulsar Her X-1 observed with the INTEGRAL satellite in July-August 2005.

Methods. The data analyzed in this work cover a substantial part of one main-on state of the source. The short time scale pulse period development is measured. X-ray pulse profiles for different energy ranges and time intervals are constructed. Pulse-averaged and pulse-phase resolved broad band X-ray spectra are studied. Spectral changes during X-ray dips are explored.

Results. The X-ray pulse profiles are found to change significantly during the period of observations. For the first time a strong spinup is measured within one $35 \mathrm{~d}$ cycle. Spectral characteristics observed during the X-ray dips are consistent with their interpretation as being due to partial covering as has been reported by several authors. The fundamental cyclotron absorption line is firmly observed in both pulse-averaged and pulse-phase resolved X-ray spectra. The energy, width, and the depth of the line are found to vary significantly with pulse phase.
\end{abstract}

Key words. X-rays: binaries - accretion, accretion disks - stars: neutron

\section{Introduction}

Discovered in 1972 by the Uhuru satellite (Giacconi et al. 1973; Tananbaum et al. 1972), Her X-1 is one of the most intensively studied accreting pulsars. Being part of a low mass X-ray binary it shows strong variability on very different time scales: the $1.24 \mathrm{~s}$ spin period of the neutron star, the $1.7 \mathrm{~d}$ binary period, the $35 \mathrm{~d}$ period of precession of the warped and tilted accretion disk (Gerend \& Boynton 1976; Shakura et al. 1999; Ketsaris et al. 2000), and the $1.65 \mathrm{~d}$ period of the pre-eclipse dips (Giacconi et al. 1973; Tananbaum et al. 1972). Due to the high orbital inclination of the system $\left(i>80^{\circ}\right)$ the counter-orbitally precessing warped accretion disk around the neutron star covers the X-ray source from the observer during a substantial part of the $35 \mathrm{~d}$ period. This gives rise to the alternation of so-called on (high $\mathrm{X}$-ray flux) and off (low X-ray flux) states. The $35 \mathrm{~d}$ cycle contains two on states - the main-on and the short-on - separated by $\sim 7-8 \mathrm{~d}$ off state. The X-ray flux in the middle of the main-on state is $\sim 4-5$ times higher than that in the middle of the short-on. The sharp transition from the off-state to the main-on is called the turn-on of the source. Turn-ons are usually used to count the cycles. The $35 \mathrm{~d}$ period manifests itself also in variations of the shape of X-ray pulse profiles (Soong et al. 1990b; Trümper et al. 1986; Deeter et al. 1998; Scott et al. 2000) and modulation of optical light curves (Gerend \& Boynton 1976; Howarth \& Wilson 1983).

In spite of a large amount of available observational data, the physical interpretation of several observed phenomena in Her X-1 are still unclear. For example, the physical mechanisms responsible for the disk precession, the X-ray dips, and the evolution of X-ray pulse profiles are highly debated. In this work we present X-ray observations of Her X-1 performed with the INTEGRAL observatory.

In Sect. 2 we describe the observations and data processing. In Sect. 3.1 we present energy- and time-dependent pulse profiles. In Sect. 3.2 we explore the behavior of the pulse period. The spectral analysis is presented in Sects. 4.1 (pulseaveraged spectra), 4.2 (spectral changes during X-ray dips), and 4.3 (pulse-resolved analysis). The results are discussed in Sect. 5. In Sect. 6 we present a summary and conclusions.

\section{Observations and data processing}

Her X-1 was observed by INTEGRAL (Winkler et al. 2003) on July 22-August 3, 2005 (MJD: 53573-53585 ${ }^{1}$ ). The observations were spread over $\sim 5$ orbital revolutions of the satellite: revs. 338, 339, 340, 341, and 342. In our analysis we used data obtained with the instruments JEM-X (Lund et al. 2003), IBIS/ISGRI (Lebrun et al. 2003; Ubertini et al. 2003), and SPI (Vedrenne et al. 2003). We used the energy range $\sim 3-20 \mathrm{keV}$ for JEM-X and $\sim 20-100 \mathrm{keV}$ for ISGRI and SPI (the spectrum of Her X-1 falls off steeply with energy so that there is no signal above $100 \mathrm{keV}$ ). The data processing was performed with the version 6.0 Offline Science Analysis (OSA) software distributed by ISDC (Courvoisier et al. 2003). An additional gain correction based on the analysis of the position of the tungsten background line was added (similar to Tsygankov et al. 2006). For the pulse-phase resolved spectroscopy we also used the

1 MJD = JD-2 400000.5 . 

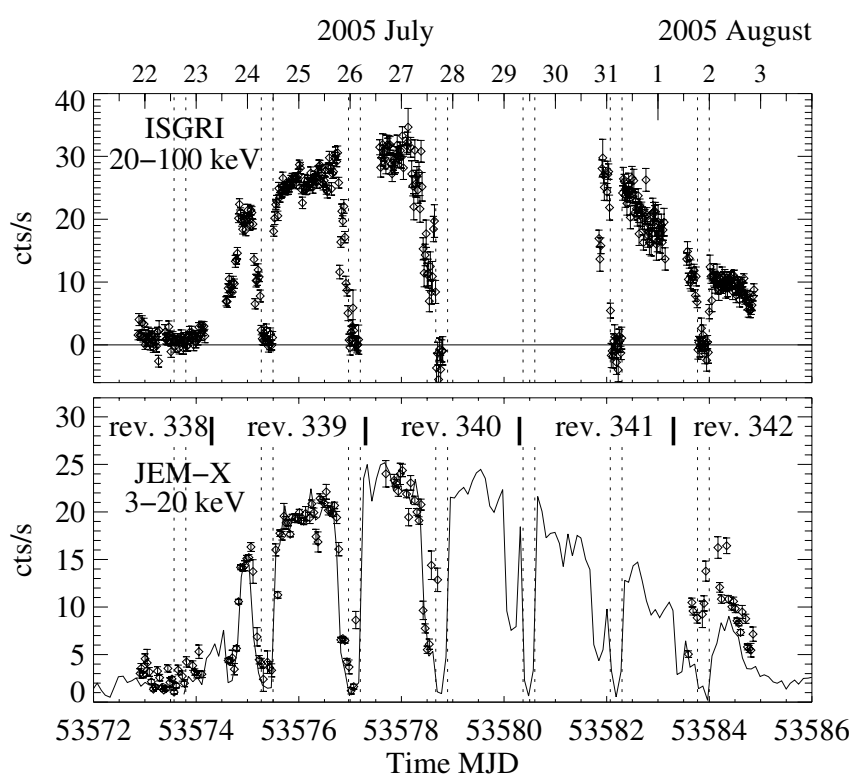

Fig. 1. X-ray light curves of Her X-1 obtained with ISGRI (top panel) and JEM-X (bottom panel). The solid curve in the bottom panel shows the ASM RXTE light curve averaged over many $35 \mathrm{~d}$ cycles (Klochkov et al. 2006). The INTEGRAL revolution numbers are indicated.

software developed at IASF, Palermo for IBIS/ISGRI² (Mineo et al. 2006; Ferrigno et al. 2007). This software allows us to construct phase-energy matrices from which pulse-resolved spectra and energy-resolved pulse profiles can easily be extracted. The results of the analysis of pulse phase averaged spectra were independently checked using the software package developed at Space Research Institute, Moscow.

Figure 1 shows the light curves of Her X-1 obtained with ISGRI (top) and JEM-X (bottom). The observations partially cover a main-on state of the source, $35 \mathrm{~d}$ phases $\phi_{\text {pre }} \sim 0-0.11$ and $\phi_{\text {pre }} \sim 0.20-0.28\left(\phi_{\text {pre }}=0\right.$ is the phase of the turn-on of the source). The turn-on occurred at MJD 53 574.7, corresponding to orbital phase $\phi_{\text {orb }} \sim 0.7$. The solid curve on top of the JEM-X light curve is the ASM RXTE (Levine et al. 1996) light curve averaged over many $35 \mathrm{~d}$ cycles and renormalized to match the one from JEM-X. All JEM-X data from revolution 341 are rejected by the ISDC team because of a strong solar flare. It is seen that the light curve is typical for Her X-1. Pre-eclipse dips (decreases of the flux just before the eclipse) are clearly seen.

\section{Timing analysis}

\subsection{Pulse profiles}

To construct the $1.24 \mathrm{~s}$ pulse profiles of the source all photon arrival times were converted to the solar system barycenter and corrected for binary motion. The orbital parameters (Table 1) used for the binary correction are based on an updated ephemeris arrived at by combining historical timing data with the timing results of the latest RXTE observations of Her X-1 (the corresponding paper is being prepared). As a folding period to construct pulse profiles we used $P_{\text {spin }}=1.23775836(10) \mathrm{s}$ which was obtained with the standard epoch folding method (Leahy et al. 1983).

It was found that the pulse profiles do not change significantly during the start (revs. 339 and 340) and during the end

\footnotetext{
2 http://www.ifc.inaf.it/ ferrigno/INTEGRALsoftware. html
}

Table 1. Orbital parameters of Her X-1 used to correct the arrival times of photons for orbital motion in the binary.

\begin{tabular}{ll}
\hline \hline$P_{\text {orb }}$ & $=1.700167233 \mathrm{~d}$ \\
$T_{\frac{\pi}{2}}$ & $=53571.982111$ (MJD) \\
$a \sin i$ & $=13.1831$ light s \\
\hline
\end{tabular}

(rev. 341) of the main-on state. Therefore, we constructed the pulse profiles separately for these two intervals without further splitting in time. We excluded the data falling inside X-ray eclipses, pre-eclipse dips or located outside the main-on state (during the off-state the data are very noisy and show only marginal sine-like pulsations). The resulting energy-resolved pulse profiles are shown in Fig. 2. It is seen that the profiles change significantly from the start to the end of the mainon state. The main peak (pulse phase $\sim 0.6-0.8$ ) is stronger and narrower at the end of the main-on. The interpulse (pulse phase $\sim 0.1-0.2$ ) almost disappears. This behavior is typical for Her X-1: analogous pulse profiles were observed by RXTE at different times (see e.g. Kuster et al. 2005). We also studied the energy dependence of the pulse fraction $F$ determined as

$F=\frac{I_{\max }-I_{\min }}{I_{\max }+I_{\min }}$

where $I_{\max }$ and $I_{\min }$ are background-corrected count rates in the maximum and minimum of the pulse profile respectively. It is clearly seen (Fig. 3) that the pulse fraction increases with energy (there may be a saturation at energies above $\sim 30 \mathrm{keV}$, however the statistical significance is marginal). The structure of the profile is also energy-dependent, going from a triple-peaked shape at lower energies to an almost single-peaked at higher energies. The main peak becomes narrower toward higher energies. A deeper analysis of the pulse profiles including the modeling of their shape (similar to that performed in Panchenko \& Postnov 1994 but taking into account $35 \mathrm{~d}$ variation) is ongoing and will be presented elsewhere.

\subsection{Pulse period behavior}

To explore more precisely the intrinsic (not affected by the orbital motion in the binary) spin period of the neutron star $P_{\text {spin }}$ during the INTEGRAL observations we performed a phaseconnection analysis similar to Ferrigno et al. (2007); Deeter et al. (1981) using well defined average pulse profiles. The length of the time intervals used to produce a single pulse profile was 3-5 INTEGRAL Science Windows (6-10 ks). This allowed us to produce pulse profiles of a quality sufficient to measure their shift in pulse phase with respect to other pulse profiles within $\sim 0.01 \mathrm{~s}$ uncertainties. Figure 4 shows the observed pulse arrival times minus the calculated times (using a constant pulse period). If we assign the same statistical uncertainty of $0.01 \mathrm{~s}$ (determined from the scattering of data points) to each data point then the linear fit to the data corresponding to a constant pulse period (dashed line) gives $\chi_{\text {red }}^{2}=1.9$ for 23 d.o.f. while the quadratic fit corresponding to the presence of a non-zero $\dot{P}_{\text {spin }}$ results in $\chi_{\text {red }}^{2}=1.0$ for 22 d.o.f. We conclude, therefore, that the neutron star is spinning up with $-\dot{P}_{\text {spin }}=(5.8 \pm 1.5) \times 10^{-13} \mathrm{~s} / \mathrm{s}$. This value is significantly higher than the averaged spin-up trend of Her X-1 which is $\sim 1.1 \times 10^{-13} \mathrm{~s} / \mathrm{s}$. Around the average spin-up strong variability of the pulse period from one main-on to the next is known to exist (see e.g. Fig. 1 in Staubert et al. 2006). 


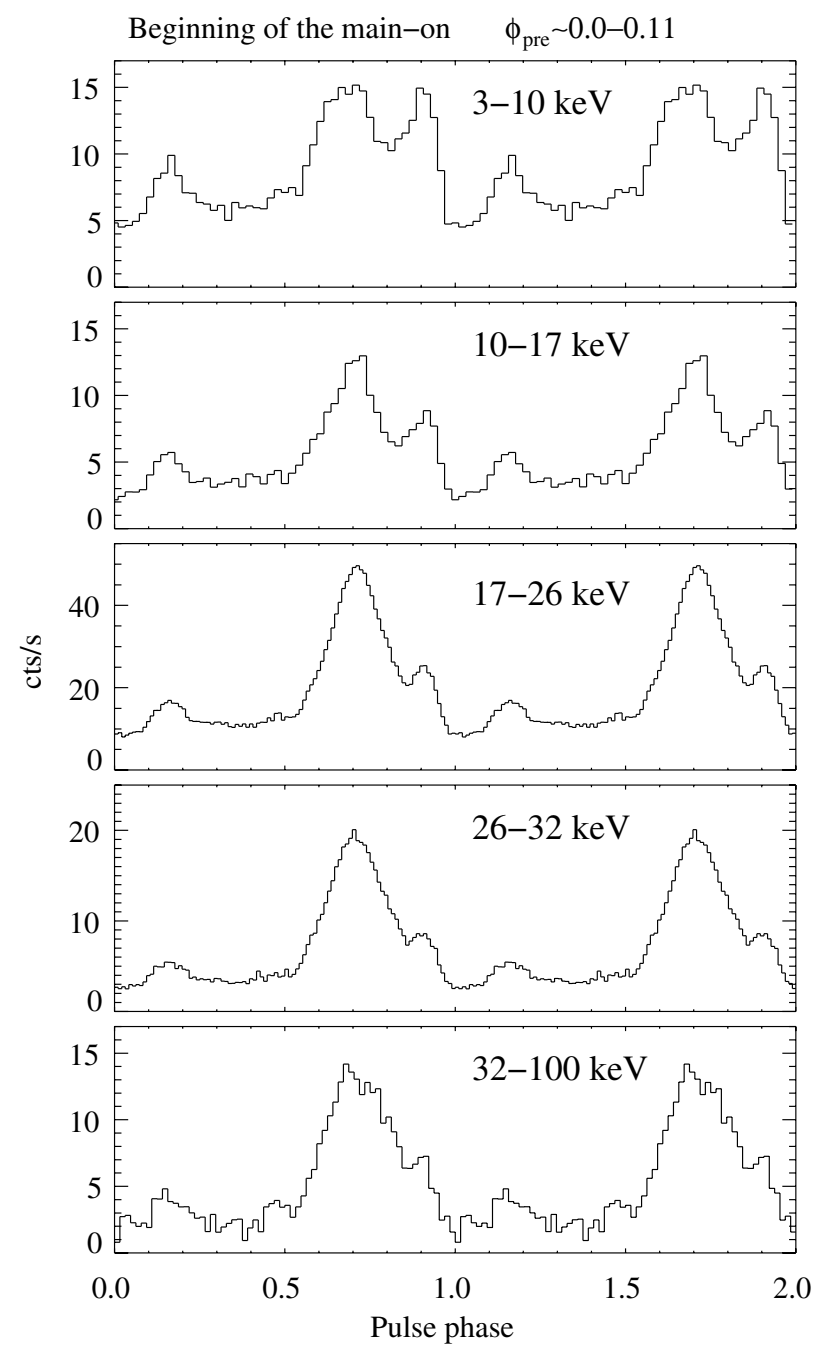

End of the main-on

$\phi_{\text {pre }} \sim 0.20-0.28$

No JEM-X data
due to a solar flare
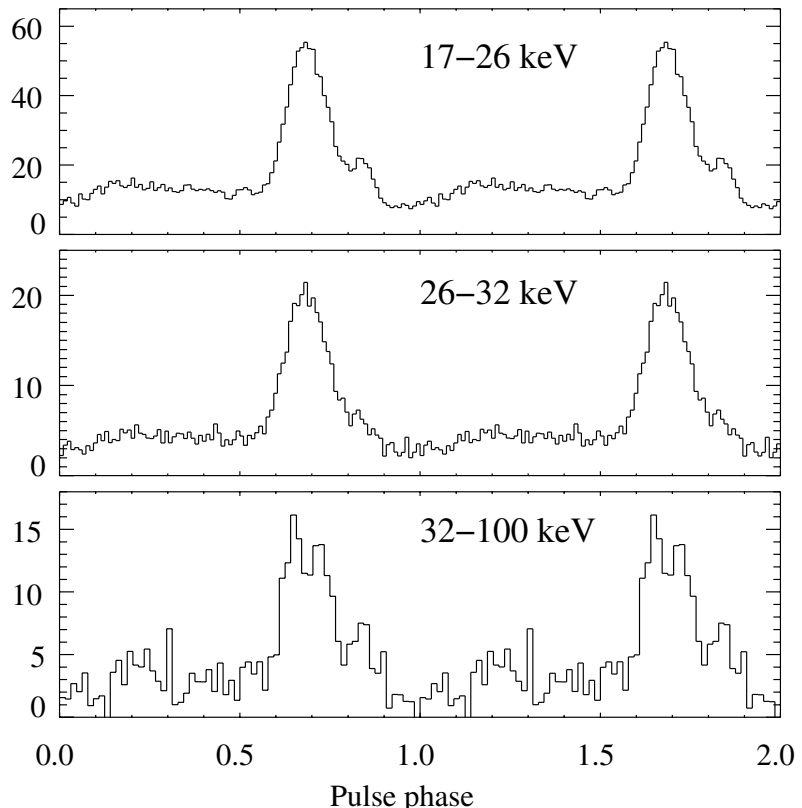

Fig. 2. Energy-resolved X-ray pulse profiles of Her X-1 at the start of the main-on (left column) and at the end of the main-on (right column).

\section{Spectral analysis}

The spectral analysis was performed using the XSPEC v.11.3.21 spectral fitting package (Arnaud 1996). For the start of the mainon the data from all three X-ray instruments were used. The fit, however, is mainly driven by JEM-X $(\$ 20 \mathrm{keV})$ and ISGRI $(\gtrsim 20 \mathrm{keV})$ data. The spectra obtained with SPI $(\gtrsim 20 \mathrm{keV})$ have much lower statistical significance. For the end of the main-on only the higher energy part of the spectrum is available since the $J E M-X$ data could not be used due to a solar flare (see above). Following the OSA User Manuals ${ }^{3}$, we added systematic errors at a level of $2 \%$ in quadrature to all JEM-X and ISGRI spectral points in order to account for uncertainties in the response matrices of the respective instruments.

\subsection{Pulse-averaged spectra}

To construct the pulse-averaged spectrum, we used the data from revolutions 339 and 340 (the start of the main-on state, see Fig. 1). During this interval the data from all three X-ray instruments are available. Eclipses and dips, which are clearly seen in the light curve (Fig. 1) and have sharp boundaries, were excluded from the analysis. To model the broad band spectral

${ }^{3}$ http://isdc.unige.ch/index.cgi?Support+documents continuum we used a power law with the exponential cutoff model highecut of XSPEC:

$I_{\text {cont }} \propto \begin{cases}E^{-\Gamma}, & \text { if } E \leq E_{\text {cutoff }} \\ E^{-\Gamma} \cdot \exp \left(-\frac{E-E_{\text {cutoff }}}{E_{\text {fold }}}\right), & \text { if } E>E_{\text {cutoff }}\end{cases}$

where $E$ is the photon energy; $\Gamma, E_{\text {cutoff }}$, and $E_{\text {fold }}$ are model parameters. A Gaussian line is added to model the iron fluorescence line at $\sim 6.5 \mathrm{keV}$. The cyclotron absorption feature is modeled by a multiplicative absorption line with a Gaussian optical depth profile. So, the final spectral function $I$ is the following:

$$
\begin{aligned}
I= & I_{\text {cont }} \cdot \exp \left\{-\tau_{\text {cycl }} \exp \left(-\frac{\left(E-E_{\text {cycl }}\right)^{2}}{2 \sigma_{\text {cycl }}^{2}}\right)\right\} \\
& +K \exp \left(-\frac{\left(E-E_{\mathrm{Fe}}\right)^{2}}{2 \sigma_{\mathrm{Fe}}^{2}}\right),
\end{aligned}
$$

where $E_{\text {cycl }}$ and $\sigma_{\text {cycl }}$ are the centroid energy and width of the cyclotron line, $E_{\mathrm{Fe}}$ and $\sigma_{\mathrm{Fe}}$ are the energy and width of the iron emission line. $\tau_{\mathrm{cycl}}$ and $K$ are the numerical constants describing the strength of the cyclotron line and iron line, respectively. The count rate spectrum is shown in Fig. 5. The middle panel shows the residuals after fitting the spectrum with the continuum model without the iron emission line and the cyclotron absorption line. Systematic features around $6 \mathrm{keV}$ and $40 \mathrm{keV}$ are clearly seen. 


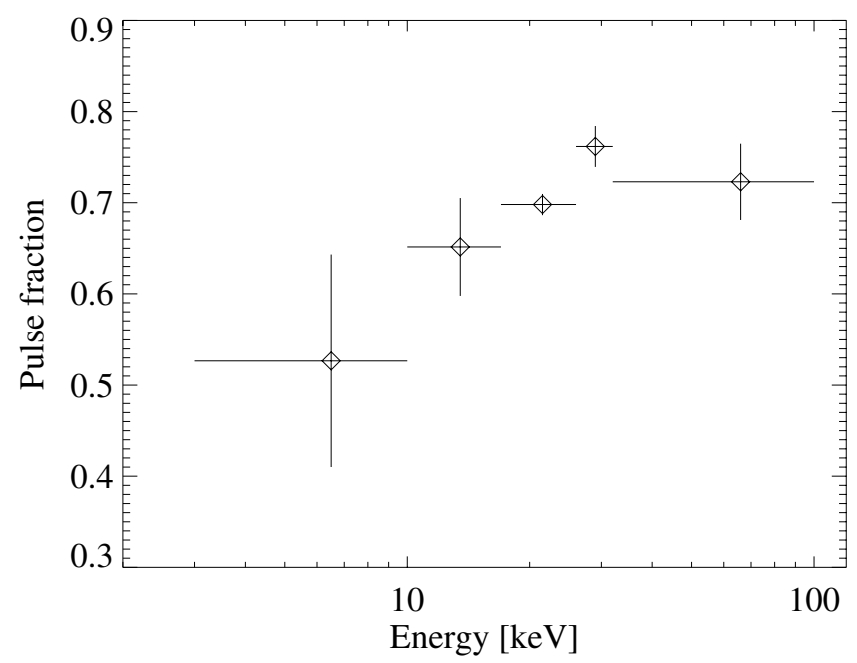

Fig. 3. Pulse fraction of Her X-1 as a function of energy.

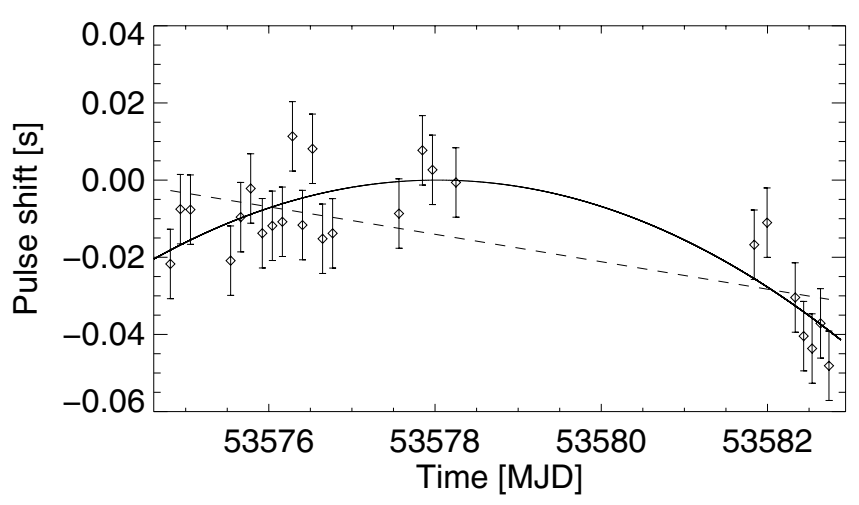

Fig. 4. Observed pulse arrival times of Her X-1 minus calculated times using a constant pulse period. The dashed line shows a linear fit corresponding to a constant pulse period while the solid line shows a quadratic fit corresponding to a $\dot{P}_{\text {spin }}=(-5.8 \pm 1.5) \times 10^{-13} \mathrm{~s} / \mathrm{s}$.

The bottom panel shows the residuals of our final fit which includes the two lines.

To account for large systematic uncertainties in the normalization of the instruments we introduced in our models a free multiplicative factor for each instrument: $F_{\text {ISGRI }}, F_{\text {SPI }}$ and $F_{\text {JEM-X }}$ (for $J E M-X$ the factor was fixed to 1.0 ). The best-fit parameters with corresponding $1 \sigma(68 \%)$-uncertainties are listed in Table 2 . The position of the cyclotron line at $38.2_{-0.6}^{+0.8} \mathrm{keV}$ is consistent with that measured by RXTE at a similar time (see Table 1 in Staubert et al. 2007b) and, therefore, supports the correlation between the cyclotron line energy and the maximum main-on flux found by Staubert et al. (2007b).

We have checked the presence and the centroid energy of the cyclotron line using different continuum models (such as e.g. Fermi-Dirac cutoff, Tanaka 1986) as well as using different line profiles (e.g. Lorenzian profile). It was found that the presence and the energy of the feature are independent of the choice of the continuum and the line profile model. The highecut model was found to provide a better description of the spectral continuum in Her X-1 than other models. Additionally, it allows us to compare our results with previous observations of the source, most of which were analyzed using the highecut model (see e.g. Gruber et al. 2001). A discontinuity of the first derivative of the spectral function at $E_{\text {cutoff }} \sim 25 \mathrm{keV}$ (which can be noticed in the residuals in Fig. 5) is far from the cyclotron line energy
Table 2. Best fit spectral parameters of Her X-1 for the observations of revs. 339 and $340.1 \sigma(68 \%)$-uncertainties $\left(\chi_{\min }^{2}+1\right)$ for one parameter of interest are shown.

\begin{tabular}{ll}
\hline \hline Parameter & Value \\
\hline$\Gamma$ & $0.91 \pm 0.01$ \\
$E_{\text {cutoff }}[\mathrm{keV}]$ & $25.5_{-0.3}^{+0.2}$ \\
$E_{\text {fold }}[\mathrm{keV}]$ & $9.0_{-0.3}^{+0.3}$ \\
$E_{\mathrm{Fe}}[\mathrm{keV}]$ & $6.57_{-0.13}^{+0.11}$ \\
$\sigma_{\mathrm{Fe}}[\mathrm{keV}]$ & $0.50_{-0.16}^{+0.17}$ \\
$E_{\text {cycl }}$ & $38.2_{-0.6}^{+0.8}$ \\
$\sigma_{\text {cycl }}$ & $9.3_{-0.5}^{+0.4}$ \\
$\tau_{\text {cycl }}$ & $0.63_{-0.06}^{+0.04}$ \\
$F_{\text {JEM-X }}$ & $1.0($ fixed) \\
$F_{\text {ISGRI }}$ & $0.81_{-0.02}^{+0.01}$ \\
$F_{\text {SPI }}$ & $0.96 \pm 0.02$ \\
$\chi_{\text {red }}^{2} /$ d.o.f. & $1.19 / 207$ \\
\hline
\end{tabular}

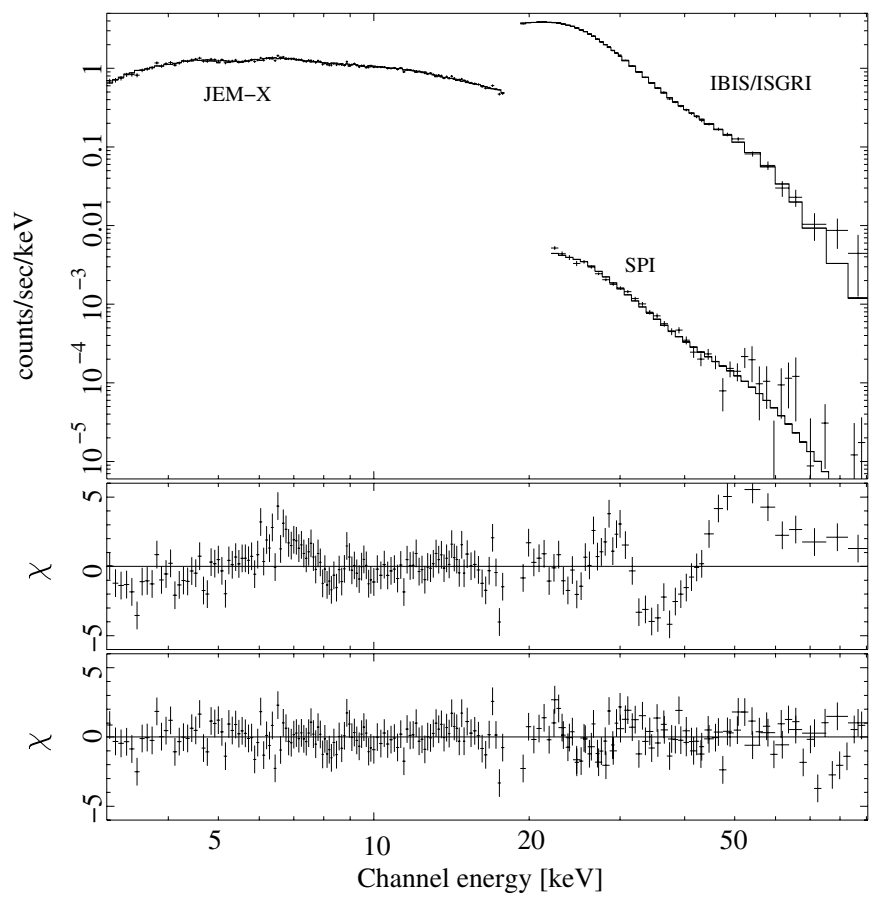

Fig. 5. Top: the pulse-averaged X-ray spectrum of Her X-1 obtained during revolutions 339 and 340 (start of the main-on); middle: residuals after fitting the spectrum with the continuum model described by Eq. (2) without the iron emission line and cyclotron absorption line (to avoid confusion the $S P I$ data are not shown in this panel, their statistical significance being lower than that of IBIS/ISGRI and JEM-X); bottom: the residuals after including the iron and cyclotron lines in the model.

and does not affect the line parameters (see however Kretschmar et al. 1997).

We also explored the pulse-averaged spectrum obtained during revolution 341 (end of the main-on). The continuum spectral parameters are poorly constrained in this case due to the absence of the low-energy ( $\$ 20 \mathrm{keV})$ part of the spectrum. However, the found position of the cyclotron line $E_{\text {cycl }}=37.3_{-1.1}^{+1.3} \mathrm{keV}$ is consistent with that measured in revolutions 339 and 340 .

\subsection{X-ray dips}

In the X-ray light curve taken with INTEGRAL one can see preeclipse dips in three subsequent orbits after the turn-on (Fig. 1). 


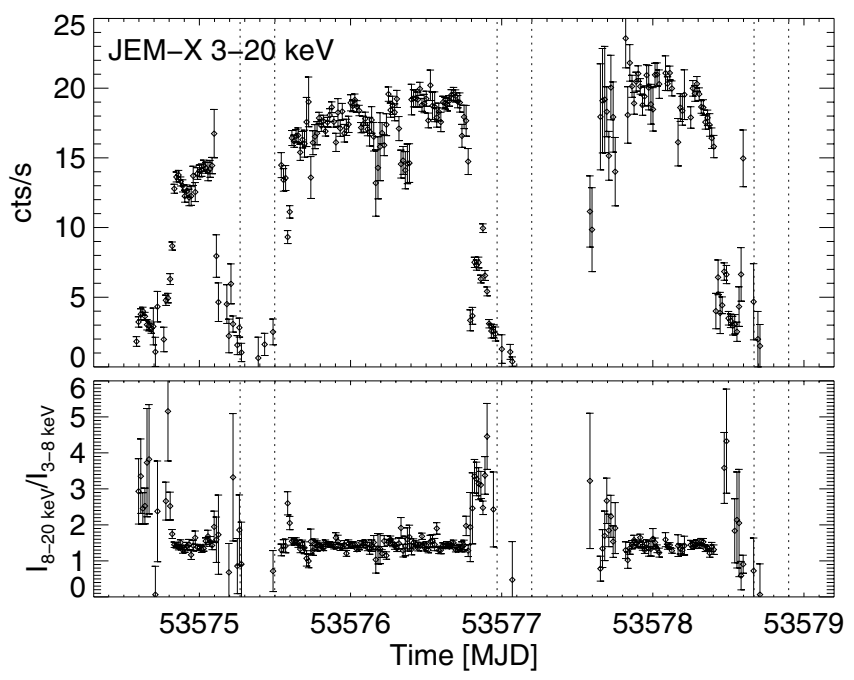

Fig. 6. The JEM-X 3-20 keV light curve (top panel) and the ratio of count rates in the harder $(8-20 \mathrm{keV})$ and softer $(3-8 \mathrm{keV})$ energy ranges as a function of time (bottom panel).

The turn-on of the source can be considered as the egress from an anomalous dip at $\phi_{\text {orb }} \sim 0.5$ (according to the model of Shakura et al. (1999) the source is partially screened by the outer accretion disk rim in both cases: during the turn-on of the source and during anomalous dips). Thus, the light curve obtained with INTEGRAL contains three pre-eclipse dips and one anomalous dip. Using the $J E M-X$ data we calculated the ratio $H=I_{8-20 \mathrm{keV}} / I_{3-8 \mathrm{keV}}$, where $I_{8-20 \mathrm{keV}}$ and $I_{3-8 \mathrm{keV}}$ are the count rates in the respective energy ranges. The ratio $H$ as a function of time is shown in the bottom panel of Fig. 6. It can be seen that $H$ increases during $\mathrm{X}$-ray dips most probably indicating low-energy absorption by the cold matter in the accretion stream (during the pre-eclipse dips) and in the outer rim of the disk (during the anomalous dip). Even though the result is dominated by the last two pre-eclipse dips, the data of the first pre-eclipse dip and the anomalous dip are consistent.

To check the absorption hypothesis we constructed JEM-X and ISGRI spectra corresponding to the four dips and compared them with those obtained outside the dips. We are aware of the fact that due to different formation mechanisms of anomalous and pre-eclipse dips their spectral characteristics might be different. But due to the low statistics we cannot perform a separate analysis of the two kinds of dips (the spectral parameters are not restricted in this case). To fit the spectrum from the dips we used the approach of Kuster et al. (2005). It was assumed that a combination of direct and absorbed radiation is observed during the dips. Thus, we used a partial covering model which combines both absorbed and non-absorbed spectra to fit the data during the dips. We used the same continuum model as in the previous section. The final spectral model can be written as

$I(E)=A \cdot\left[1+\alpha(E) \cdot I_{\text {cont }}\right]$,

where $I_{\text {cont }}$ is the continuum model described in the previous section, and

$\alpha(E)=B \cdot \mathrm{e}^{-N_{\mathrm{H}} \sigma_{\mathrm{bf}}(E)}$,

where $\sigma_{\text {bf }}(E)$ is the photoabsorption cross-section per hydrogen atom for matter of cosmic abundances (Bałucińska-Church \& McCammon 1992) used in the phabs model of XSPEC and $N_{\mathrm{H}}$ is the equivalent hydrogen column density. A larger value of $B$ implies a larger degree of absorbed flux. From the fit

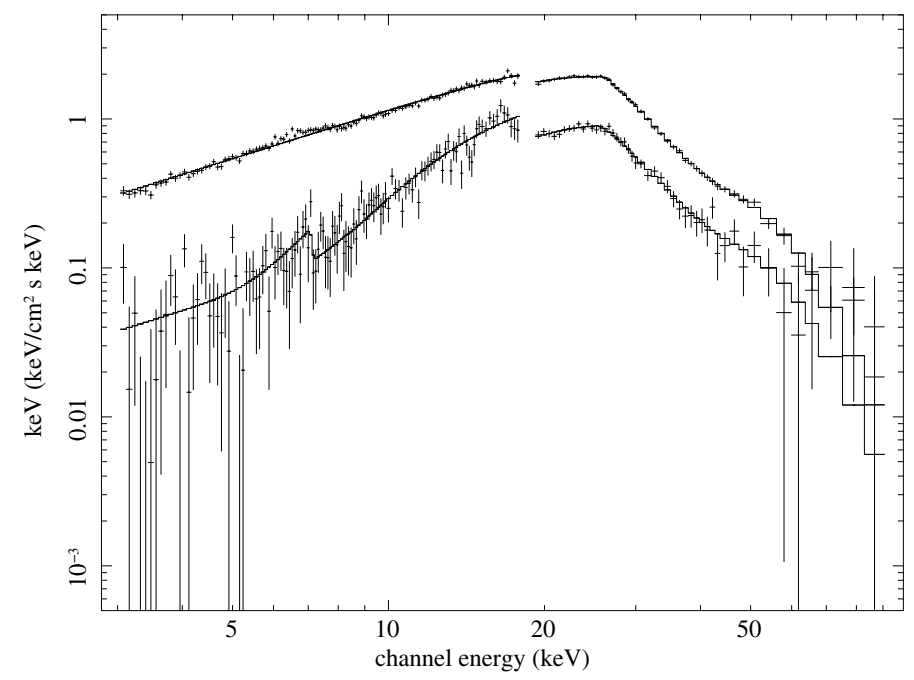

Fig. 7. Examples of pulse-averaged spectra of Her X-1 from the dips (lower curve) and outside the dips (upper curve). The difference of the spectral shape at lower energies caused by photo-absorption inside the dips is clearly seen. The step-like feature at $\sim 7 \mathrm{keV}$ in the spectrum of the dips corresponds to the iron K-edge (as implemented in the phabs model of XSPEC).

of the spectrum of the dips we found $B=0.24 \pm 0.03$ and $N_{\mathrm{H}}=\left(111_{-19}^{+18}\right) \times 10^{22} \mathrm{~cm}^{-2}$. The best-fit parameters of the spectral continuum $I_{\text {cont }}$ are consistent with those found from fitting the spectrum outside the dips (Table 2). To compare this result with the spectrum outside the dips, the latter was also fit by the partial covering model (Eq. (4)). Both spectra (inside and outside the dips) are shown in Fig. 7. For the spectrum outside the dips (the upper one) the $N_{\mathrm{H}}$ parameter was found to be consistent with zero and the $B$ factor, consequently, was not restricted. We conclude therefore, that the X-ray data of Her X-1 during the dips are consistent with the partial absorption model.

\subsection{Pulse-phase-resolved spectra}

It is well known that the X-ray spectrum of Her X-1 varies with 1.24 s pulse phase (Voges et al. 1982; Voges 1985; Soong et al. 1990a). So, we have performed a separate analysis of the spectra accumulated in different pulse phase intervals. Figure 8 shows an example of pulse resolved spectra of the source. Variability of the continuum and the cyclotron line is clearly seen.

Since the shape of the pulse profile changes significantly from the start of the main-on (revs. 339, 340) to its end (rev. 341), we analyzed the data from these two intervals separately. Phase binning in each case was chosen to provide similar statistics in each spectral bin. Pulse phase zero is the same as in Fig. 2. The $\mathrm{X}$-ray spectrum of each phase bin was fitted with the spectral model described in Sect. 4.1. Figures 9 and 10 show the best-fit spectral parameters as a function of pulse phase for revs. 339 and 340 (left) and the rev. 341 (right). Vertical error bars correspond to $1 \sigma(68 \%)$-uncertainties. The dotted line shows the corresponding pulse profile. The spectral parameters in different pulse phase bins are also listed in Table 3. Since no JEM-X data are available for rev. 341 the power law photon index $\Gamma$ could not be restricted by the fit in this case. The iron line at $\sim 6.5 \mathrm{keV}$ was detected only in the phase bin $0.1-0.54$, corresponding to the off-pulse interval. The data from other phase intervals do not require inclusion of the line in the spectral model to obtain a good fit. For the data corresponding to revs. 339 and 340, it is seen that all continuum 


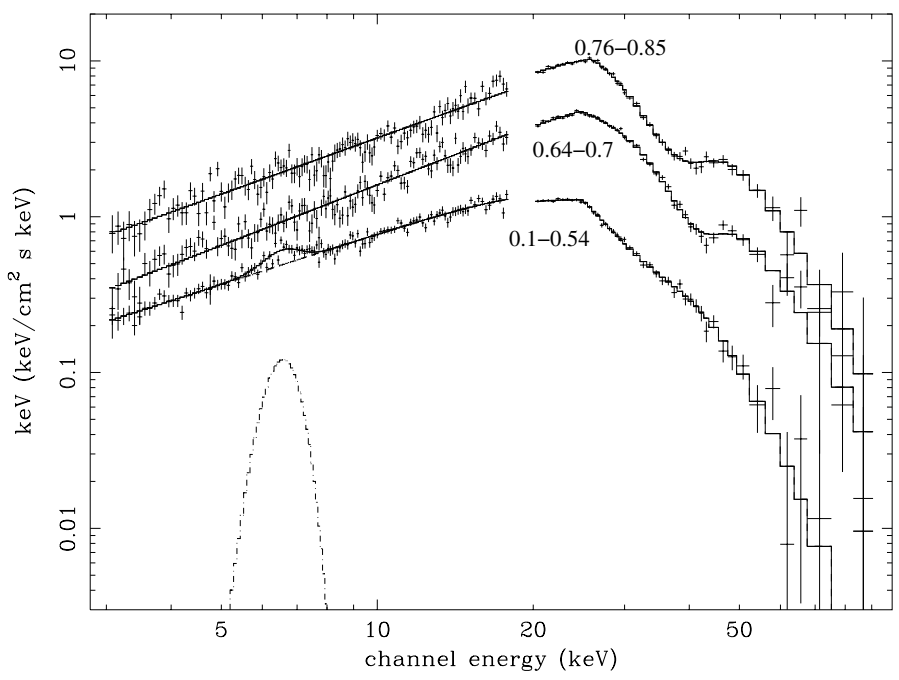

Fig. 8. Examples of pulse-resolved X-ray spectra of Her X-1 during revolutions 339 and 340. Pulse phase intervals are indicated (the pulse phase zero is the same as in Fig. 2).

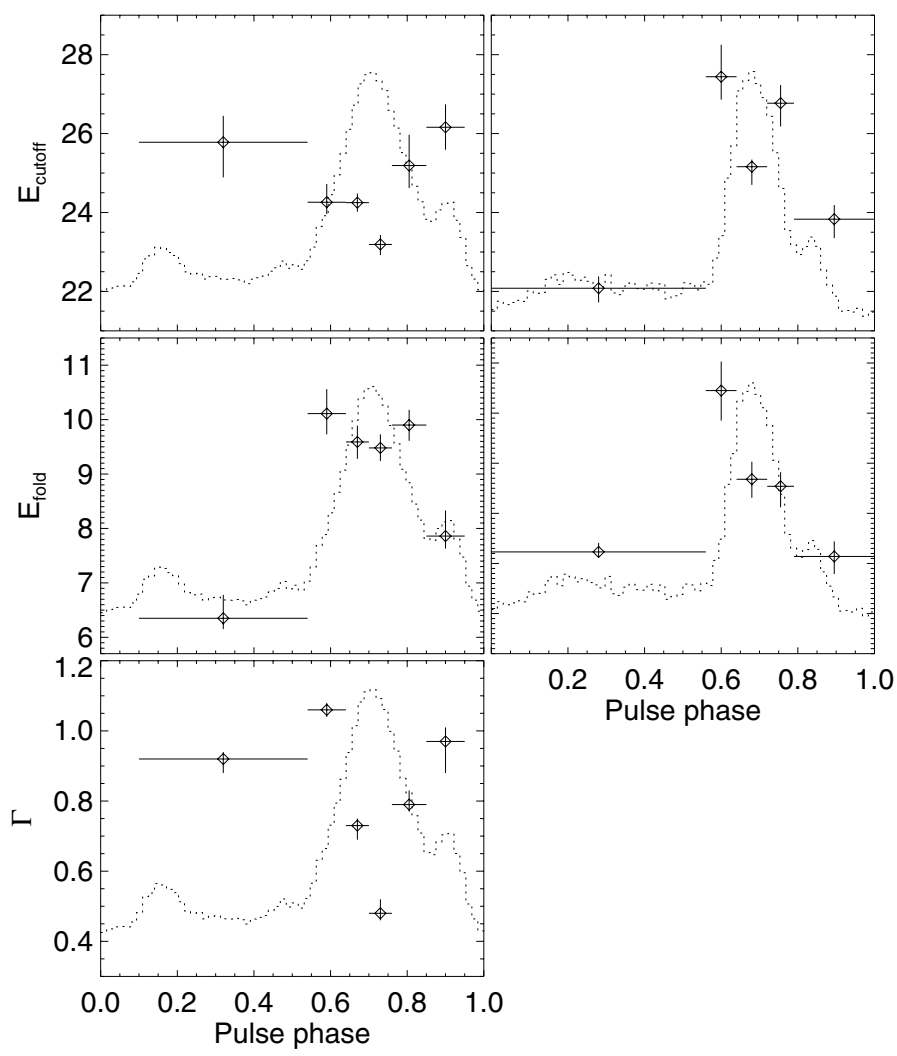

Fig. 9. Best-fit spectral continuum parameters as functions of the pulse phase for revolutions 339 and 340 (left) and the revolution 341 (right). The dashed curve shows the corresponding pulse profile. Vertical error bars correspond to $1 \sigma(68 \%)$-uncertainties.

parameters and the cyclotron line parameters are highly variable with pulse phase. For revolution 341 the picture is not so clear due to the absence of the low-energy $(\$ 20 \mathrm{keV})$ part of the spectrum. Also the total exposure time is shorter and the average flux of the source in rev. 341 is less than those during revolutions 339 and 340 .

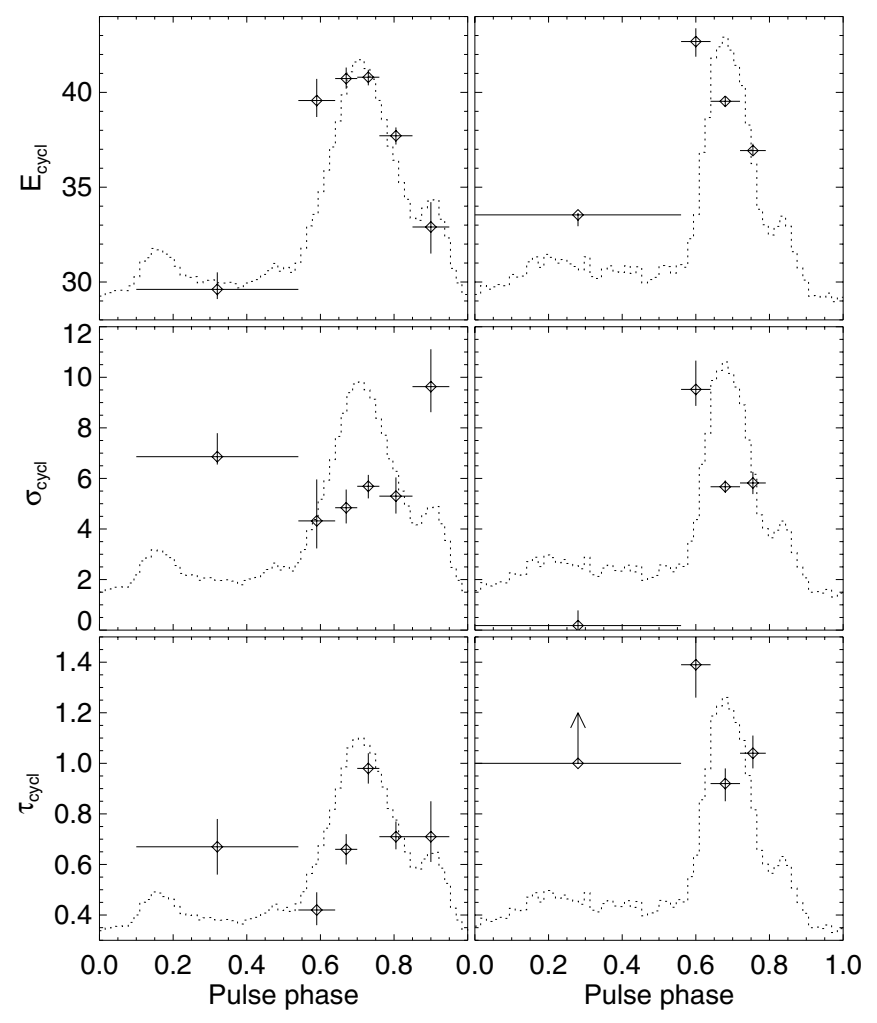

Fig. 10. The same as in Fig. 9 but for the cyclotron line parameters (left: revolutions 339 and 340; right: revolution 341).

\section{Discussion}

\subsection{Pulse profiles and pulse period}

In Sect. 3.1 we constructed $1.24 \mathrm{~s}$ X-ray pulse profiles of Her X-1. The shape of the profiles is both energy- and timedependent. At higher energies the main peak becomes narrower and the pulsed fraction increases (Figs. 2 and 3). Such a dependence of the profile on energy is typical of accreting pulsars (see e.g. Tsygankov et al. 2007). It can be understood in a simple purely geometrical picture: if the rotation axis of the neutron star is inclined with respect to the axis of its magnetic field, it is expected that the upper part of the accretion column emitting softer photons is seen during a larger part of the neutron star spin period while the emission region of harder photons, the "base" of the accretion column, is screened by the neutron star surface during most of the spin period. Additionally, the emission diagram of harder photons most probably originating close to the base of the accretion column is believed to be narrower than that of softer photons due to the strong dependence of the scattering cross-section on the angle between the direction of a photon and the magnetic field lines (see e.g. Basko \& Sunyaev 1976).

The difference of the pulse profiles in the left and right columns of Fig. 2 (corresponding to the start and the end of the main-on state, respectively) shows the evolution of the profiles with $35 \mathrm{~d}$ phase. Two models have been proposed to explain these variations. Scott et al. (2000) suggested that the change of the profile from the start to the end of the main-on state is due to a "resolved occultation" of the emitting regions on the neutron star by the precessing accretion disk. According to this model, the disk progressively occults the neutron star towards the end of the main-on. The other model assumes free precession of the neutron star (Trümper et al. 1986; Kahabka 1987, 1989; Prokhorov et al. 1990; Shakura et al. 1998). In this model the observed 
Table 3. Best fit spectral parameters of Her X-1 in different pulse phase intervals. $1 \sigma(68 \%)$-uncertainties $\left(\chi_{\min }^{2}+1\right)$ for one parameter of interest are shown.

\begin{tabular}{lllllll}
\hline \hline Phase bin & $\Gamma$ & $E_{\text {cutoff }}$ & $E_{\text {fold }}$ & $E_{\text {cycl }}$ & $\sigma_{\text {cycl }}$ & $\tau_{\text {cycl }}$ \\
\hline Revs. 339 and 340 & & & & & & \\
$0.10-0.54$ & $0.92_{-0.04}^{+0.02}$ & $25.8_{-0.9}^{+0.7}$ & $6.35_{-0.20}^{+0.43}$ & $29.6_{-0.5}^{+0.9}$ & $6.86_{-0.31}^{+0.93}$ & $0.67_{-0.11}^{+0.11}$ \\
$0.54-0.64$ & $1.06_{-0.02}^{+0.02}$ & $24.3_{-0.3}^{+0.5}$ & $10.11_{-0.38}^{+0.45}$ & $39.6_{-0.9}^{+1.1}$ & $4.32_{-1.09}^{+1.64}$ & $0.42_{-0.06}^{+0.07}$ \\
$0.64-0.70$ & $0.73_{-0.04}^{+0.02}$ & $24.3_{-0.2}^{+0.2}$ & $9.59_{-0.31}^{+0.30}$ & $40.7_{-0.5}^{+0.6}$ & $4.84_{-0.62}^{+0.72}$ & $0.66_{-0.06}^{+0.06}$ \\
$0.70-0.76$ & $0.48_{-0.02}^{+0.04}$ & $23.2_{-0.3}^{+0.2}$ & $9.48_{-0.24}^{+0.25}$ & $40.8_{-0.4}^{+0.4}$ & $5.69_{-0.48}^{+0.45}$ & $0.98_{-0.06}^{+0.06}$ \\
$0.76-0.85$ & $0.79_{-0.02}^{+0.04}$ & $25.2_{-0.6}^{+0.0}$ & $9.90_{-0.29}^{+0.28}$ & $37.7_{-0.5}^{+0.4}$ & $5.30_{-0.69}^{+0.74}$ & $0.71_{-0.05}^{+0.06}$ \\
$0.85-0.95$ & $0.97_{-0.09}^{+0.04}$ & $26.2_{-0.6}^{+0.6}$ & $7.86_{-0.23}^{+0.47}$ & $32.9_{-1.4}^{+1.3}$ & $9.63_{-1.01}^{+1.48}$ & $0.71_{-0.10}^{+0.14}$ \\
\hline Rev. 341 & & & & & & \\
$0.00-0.56$ & - & $22.1_{-0.4}^{+0.3}$ & $8.23_{-0.11}^{+0.18}$ & $33.5_{-0.6}^{+0.1}$ & $0.18_{-0.17}^{+0.60}$ & $\geq 1.0$ \\
$0.56-0.64$ & - & $27.4_{-0.6}^{+0.8}$ & $11.45_{-0.60}^{+0.58}$ & $42.7_{-0.8}^{+0.7}$ & $9.52_{-0.65}^{+1.14}$ & $1.39_{-0.13}^{+0.13}$ \\
$0.64-0.72$ & - & $25.2_{-0.5}^{+0.2}$ & $9.68_{-0.37}^{+0.35}$ & $39.5_{-0.3}^{+0.3}$ & $5.67_{-0.23}^{+0.25}$ & $0.92_{-0.07}^{+0.06}$ \\
$0.72-0.79$ & - & $26.8_{-0.6}^{+0.5}$ & $9.54_{-0.42}^{+0.28}$ & $36.9_{-0.2}^{+0.4}$ & $5.82_{-0.44}^{+0.44}$ & $1.04_{-0.06}^{+0.07}$ \\
$0.79-1.00$ & - & $23.8_{-0.5}^{+0.4}$ & $8.14_{-0.35}^{+0.30}$ & - & - & - \\
\hline
\end{tabular}

behavior of the profile is explained assuming that the emission region on the star surface has a complex shape (due to the presence of higher multipole components of the neutron star's magnetic field). Changing the viewing conditions of the emitting region with the phase of the free precession causes the observed variation of the pulse profile (Ketsaris et al. 2000; Wilms et al. 2003). In pulse profiles obtained with INTEGRAL one can see that the count rate at the maximum of the main peak is higher at the end of the main-on than that during the start of the main-on (Fig. 2). In the case of a progressive occultation one would only expect a decrease in the intensity of any feature in the profile towards the end of the main-on. In the model of free precession, however, viewing conditions of different parts of the emission region may change with the precessional phase of the star in such a way that the intensity of particular features of the profile (e.g. of the main peak) will increase towards the end of the main-on. We argue, therefore, that the INTEGRAL observations analyzed in this work support the model of a freely precessing neutron star as an explanation of the time variation of the pulse profile in Her X-1.

In Sect. 3.2 we determined the time derivative of the intrinsic (not affected by the orbital motion in the binary) pulse period of the neutron star in Her X-1 during the INTEGRAL observation. The pulsar was found to spin-up with the rate $-\dot{P}_{\text {spin }}=(5.8 \pm 1.5) \times 10^{-13} \mathrm{~s} / \mathrm{s}$. This value is more than five times larger than the mean spin-up trend of Her X-1 $\left(\sim 1.1 \times 10^{-13} \mathrm{~s} / \mathrm{s}\right)$ and consistent with the variations of $P_{\text {spin }}$ between subsequent 35 d cycles, e.g. as seen by BATSE (Kunz 1996; Nagase 1989; Sunyaev et al. 1988) and RXTE (Staubert et al. 2006). However, this is the first time that such strong pulse period variations have been measured within one $35 \mathrm{~d}$ cycle. A deeper analysis of these pulse period variations and their corelation with the X-ray luminosity (including the data from INTEGRAL presented here) is currently being performed and will be presented in a separate paper.

\subsection{Absorption during $X$-ray dips}

In Sect. 4.2 we have shown that the X-ray spectrum of Her X-1 obtained during X-ray dips can be modeled using a partial covering model which assumes that the observed spectrum is a combination of direct and absorbed radiation. Such a combination has already been observed in Her X-1 during X-ray turnons (Davison \& Fabian 1977; Becker et al. 1977; Parmar et al. 1980; Kuster et al. 2005) and also during X-ray dips (Vrtilek \& Halpern 1985, although the observations included only the lower energy part of the spectrum $\$ 20 \mathrm{keV}$ ).

According to the model of Shakura et al. (1999) and Klochkov et al. (2006) (which we adopt in this work), most of the $\mathrm{X}$-ray dips are produced by the occultation of the X-ray source by the cold matter in the accretion stream which moves out of the system's orbital plane. If the stream is not homogeneous but consists of "blobs" of material then the source will be screened during some time intervals within a dip. Between these intervals the source will be visible or seen through less dense material. This picture seems to be confirmed by the complicated behavior of the light curve inside the dips (Fig. 6). In our analysis, in order to construct an X-ray spectrum of acceptable quality we had to accumulate the flux during complete dips. The spectrum in this case will contain a superposition of the absorbed and nonabsorbed flux. The partial covering model clearly fits the spectrum of the dips better than the simple absorption model.

\subsection{Pulse-phase variability of the $X$-ray spectrum}

The dependence of the spectral parameters in Her X-1 on pulse phase observed by INTEGRAL confirms the results obtained earlier with HEAO-1 (Soong et al. 1990b) but provide better restriction of continuum parameters. The spectral variation with pulse phase is a common feature in X-ray pulsars (see e.g. Kreykenbohm et al. 2004, and references therein) which is usually attributed to the change of the viewing angle of the accretion region on the neutron star surface. Below we show that the pulse-phase variability observed in Her X-1 is consistent with this interpretation.

As one can see in Fig. 9, the power law photon index $\Gamma$ decreases during the main peak. It ranges from $\sim 1.0$ in the offpulse to $\sim 0.5$ close to the maximum of the main peak (see also Lutovinov et al. 2000). This effect reflects the sharpening of the main peak with energy which is observed in energy-resolved pulse profiles constructed in Sect. 3.1 (Fig. 2). The spectral hardening in the main peak can be explained by the dependence of 
the optical depth on the angle between the line-of-sight and the magnetic field lines (Pravdo et al. 1977). The closer the viewing direction is to the magnetic axis, the deeper we look into the emission region where harder photons originate. Since it is generally accepted that the main peak corresponds to the radiation from one of the two magnetic poles, the viewing direction is closest to the magnetic axis during the main peak which causes the observed effect.

The exponential folding energy $E_{\text {fold }}$ is much higher during the main peak than during the rest of the pulsation cycle. In Comptonized X-ray spectra (which are normally observed from accreting pulsars) this parameter is believed to be proportional to the plasma temperature (see e.g. Rybicki \& Lightman 1979). As already mentioned, during the main peak we see the emission from higher optical depth. This means that the observed radiation originates closer to the base of the accretion column where the plasma temperature is higher.

The cyclotron line centroid energy $E_{\text {cycl }}$ is also higher during the main peak of the profile (Fig. 10). The relative amplitude of its variation is $\sim 25 \%$. As argued from $\Gamma$ and $E_{\text {fold }}$, during the main peak we see X-rays that originate closer to the neutron star surface, i.e. where the magnetic field strength is higher. The increase in $E_{\text {cycl }}$ during the main peak is, therefore, qualitatively consistent with this picture. If one assumes that the radiation comes from a compact accretion column located at the magnetic pole of the neutron star with a pure dipole field then the change in the height of the observed emission region necessary to produce a $\sim 25 \%$ variation of the magnetic field strength is $\sim 1.1 \mathrm{~km}$ (for a neutron star radius of $10 \mathrm{~km}$ ). However, as it was shown by Staubert et al. (2007a), the height of the emission and line forming region in case of Her X-1 is most probably much smaller, $\sim 10^{4} \mathrm{~cm}$. Thus, a changing height of the emitting region above the neutron star cannot explain the observed variability of the cyclotron line energy with pulse phase in this source.

Another possibility for the line energy variability is to assume a complicated structure of the magnetic field at the site of X-ray emission. As shown by Shakura et al. (1991) and Panchenko \& Postnov (1994), the complex shape of the observed pulse profiles in Her X-1 suggests that such a complicated field structure including higher multipole components is indeed present in the source. In this case, at different rotational phases of the neutron star we will observe emission coming from the regions corresponding to different sub-structures of the non-dipole magnetic field with very different field strengths. Furthermore, the numerical modeling of Her X-1 pulse profiles observed with EXOSAT performed by Panchenko \& Postnov (1994) suggests that the magnetic field stength at the ring-like structure on the neutron star's surface corresponding to the quadrupole component of the field (which was introduced in the model) is indeed lower by $\sim 27 \%$ than that at the magnetic pole. Thus, a complicated magnetic field structure on the surface of the neutron star can cause the observed $25 \%$ variation of $E_{\text {cycl }}$ with pulse phase.

\section{Summary and conclusion}

In this work we analyzed the INTEGRAL observations of the accreting X-ray pulsar Her X-1. X-ray pulse profiles of the source are constructed and their energy- and time-dependence are discussed. A strong spin-up is found during the INTEGRAL observations. This is the first time that pulse period variation is measured within one main-on state of the source. The value of the spin-up rate is consistent with the typical difference between the values of the pulse period previously found in adjacent $35 \mathrm{~d}$ cycles. Spectral changes during X-ray dips are studied. The X-ray spectrum of the dips was modeled by a partial covering model which assumes that the observed spectrum is a combination of direct and absorbed radiation. This is in agreement with the previously suggested interpretation assuming that the obscuring matter (accretion stream) is not homogeneous but rather consists of blobs of material (see e.g. Vrtilek \& Halpern 1985). Energyresolved X-ray pulse profiles as well as the variation of cyclotron line and continuum parameters with 1.24 s pulse phase were explored. The spectral changes with pulse phase are shown to be qualitatively (for the cyclotron line energy - also quantitatively) consistent with those expected from the viewing conditions of the complex emitting region which vary with the rotational phase of the neutron star.

Acknowledgements. This research is based on observations with INTEGRAL, an ESA project with instruments and science data centre funded by ESA member states (especially the PI countries: Denmark, France, Germany, Italy, Switzerland, Spain), Czech Republic and Poland, and with the participation of Russia and the USA. The work was supported by the DFG grants Sta 173/31-2 and 436 RUS 113/717/0-1 and the corresponding RBFR grants RFFI-NNIO-0302-04003 and RFFI 06-02-16025, as well as DLR grant 50 0R 0302 and RFFI 07-02-01051. We also thank ISSI (Bern, Switzerland) for hospitality during the team meetings of our collaboration. AL acknowledges the financial support of the Russian Science Support Foundation.

\section{References}

Arnaud, K. A. 1996, in Astronomical Data Analysis Software and Systems V, ed. G. H. Jacoby, \& J. Barnes, ASP Conf. Ser., 101, 17

Bałucińska-Church, M., \& McCammon, D. 1992, ApJ, 400, 699

Basko, M. M., \& Sunyaev, R. A. 1976, MNRAS, 175, 395

Becker, R. H., Boldt, E. A., Holt, S. S., et al. 1977, ApJ, 214, 879

Courvoisier, T. J.-L., Walter, R., Beckmann, V., et al. 2003, A\&A, 411, L53

Davison, P. J. N., \& Fabian, A. C. 1977, MNRAS, 178, 1P

Deeter, J. E., Pravdo, S. H., \& Boynton, P. E. 1981, ApJ, 247, 1003

Deeter, J. E., Scott, D. M., Boynton, P. E., et al. 1998, ApJ, 502, 802

Ferrigno, C., Segreto, A., Santangelo, A., et al. 2007, A\&A, 462, 995

Gerend, D., \& Boynton, P. E. 1976, ApJ, 209, 562

Giacconi, R., Gursky, H., Kellogg, E., et al. 1973, ApJ, 184, 227

Gruber, D. E., Heindl, W. A., Rothschild, R. E., et al. 2001, ApJ, 562, 499

Howarth, I. D., \& Wilson, B. 1983, MNRAS, 202, 347

Kahabka, P. 1987, NASA STI/Recon Technical Report N, 88, 19405

Kahabka, P. 1989, in ESA SP-296: Two Topics in X-Ray Astronomy, Vol. 1:

X Ray Binaries, Vol. 2: AGN and the X Ray Background, ed. J. Hunt, \& B. Battrick, 447

Ketsaris, N. A., Kuster, M., Postnov, K., et al. 2000, in Proc. Int. Workshop Hot Points in Astrophysics, JINR, Dubna, 192, ed. V. Belyaev

[arXiv: astro-ph/0010035]

Klochkov, D. K., Shakura, N. I., Postnov, K. A., et al. 2006, Astron. Lett., 32, 804

Kretschmar, P., Kreykenbohm, I., Wilms, J., et al. 1997, in ESA Special Publication, The Transparent Universe, ed. C. Winkler, T. J.-L. Courvoisier, \& P. Durouchoux, 382, 141

Kreykenbohm, I., Wilms, J., Coburn, W., et al. 2004, A\&A, 427, 975

Kunz, M. 1996, Ph.D. Thesis, Germany, University of Tübingen

Kuster, M., Wilms, J., Staubert, R., et al. 2005, A\&A, 443, 753

Leahy, D. A., Elsner, R. F., \& Weisskopf, M. C. 1983, ApJ, 272, 256

Lebrun, F., Leray, J. P., Lavocat, P., et al. 2003, A\&A, 411, L141

Levine, A. M., Bradt, H., Cui, W., et al. 1996, ApJ, 469, L33

Lund, N., Budtz-Jørgensen, C., Westergaard, N. J., et al. 2003, A\&A, 411, L231

Lutovinov, A. A., Grebenev, S. A., Pavlinsky, M. N., \& Sunyaev, R. A. 2000, Astron. Lett., 26, 691

Mineo, T., Ferrigno, C., Foschini, L., et al. 2006, A\&A, 450, 617

Nagase, F. 1989, PASJ, 41, 1

Panchenko, I. E., \& Postnov, K. A. 1994, A\&A, 286, 497

Parmar, A. N., Sanford, P. W., \& Fabian, A. C. 1980, MNRAS, 192, 311

Pravdo, S. H., Boldt, E. A., Holt, S. S., \& Serlemitsos, P. J. 1977, ApJ, 216, L23 Prokhorov, M. E., Shakura, N. I., \& Postnov, K. A. 1990, Thirty-five-day cycle of HER X-1: Synthesised optical light curves in the model of freely processing neutron star, Tech. rep. 
Rybicki, G. B., \& Lightman, A. P. 1979, Radiative processes in astrophysics (New York: Wiley-Interscience), 393

Scott, D. M., Leahy, D. A., \& Wilson, R. B. 2000, ApJ, 539, 392

Shakura, N. I., Postnov, K. A., \& Prokhorov, M. E. 1991, SvA Lett., 17, 339

Shakura, N. I., Postnov, K. A., \& Prokhorov, M. E. 1998, A\&A, 331, L37

Shakura, N. I., Prokhorov, M. E., Postnov, K. A., \& Ketsaris, N. A. 1999, A\%A, 348,917

Soong, Y., Gruber, D. E., Peterson, L. E., \& Rothschild, R. E. 1990a, ApJ, 348, 641

Soong, Y., Gruber, D. E., Peterson, L. E., \& Rothschild, R. E. 1990b, ApJ, 348, 634

Staubert, R., Klochkov, D., \& Rodina, L. 2007a, A\&A, in preparation

Staubert, R., Schandl, S., Klochkov, D., et al. 2006, in The Transient Milky Way: A Perspective for MIRAX, ed. J. Braga, F. D'Amico, \& R. E. Rothschild, American Institute of Physics Conf. Ser., 840, 65

Staubert, R., Shakura, N. I., Postnov, K., et al. 2007b, A\&A, 465, L25

Sunyaev, R. A., Gilfanov, M. R., Churazov, E. M., et al. 1988, SvA Lett., 14, 416
Tanaka, Y. 1986, in Radiation Hydrodynamics in Stars and Compact Objects, ed. D. Mihalas, \& K.-H. A. Winkler, IAU Colloq., 891, Lecture Notes in Physics (Berlin: Springer Verlag), 255, 98

Tananbaum, H., Gursky, H., Kellogg, E. M., et al. 1972, ApJ, 174, L143

Trümper, J., Kahabka, P., Oegelman, H., Pietsch, W., \& Voges, W. 1986, ApJ, 300, L63

Tsygankov, S. S., Lutovinov, A. A., Churazov, E. M., \& Sunyaev, R. A. 2006, MNRAS, 371, 19

Tsygankov, S. S., Lutovinov, A. A., Churazov, E. M., \& Sunyaev, R. A. 2007, Astron. Lett., 33, 368

Ubertini, P., Lebrun, F., Di Cocco, G., et al. 2003, A\&A, 411, L131

Vedrenne, G., Roques, J.-P., Schönfelder, V., et al. 2003, A\&A, 411, L63

Voges, W. 1985, NASA STI/Recon Technical Report N, 85, 34112

Voges, W., Pietsch, W., Reppin, C., et al. 1982, ApJ, 263, 803

Vrtilek, S. D., \& Halpern, J. P. 1985, ApJ, 296, 606

Wilms, J., Ketsaris, N. A., Postnov, K. A., et al. 2003, Izvestiya Akademii Nauk, Ser. Fizicheskaya, 67, 310

Winkler, C., Courvoisier, T. J.-L., Di Cocco, G., et al. 2003, A\&A, 411, L1 